\title{
Getting results for hematology patients through access to the electronic health record
}

by David Wiljer, Sima Bogomilsky, Pamela Catton, Cindy Murray, Janice Stewart and Mark Minden

\section{Abstract}

Purpose: To conduct a needs assessment to identify patient and provider perceptions about providing patients with access to their electronic health record in order to develop an online system that is appropriate for all stakeholders. Methods: Malignant hematology patients were surveyed and health care providers were interviewed to identify issues and validate concerns reported in the literature. Based on the analysed data, a prototype will be designed to examine the feasibility and efficacy of providing patients with access to their electronic health record and tailored information. Results: $61 \%$ of patients reported using the internet to find health information; $89 \%$ were interested in accessing their electronic health record and 79\% stated they would benefit from educational material along with the results. Staff members viewed patient online access to the record favourably, but expressed the importance of providing the necessary patient support and education. A Web-based prototype was developed for patients to review their registration data and blood results. Conclusions: Hematology oncology patients are more interested in using the internet to monitor their clinical information than to find health information. Using the constructed prototype, the feasibility of this project is currently being tested.

\section{Background}

Patient-centred models of care, such as the Picker Institute's " 8 Dimensions", emphasize the importance of providing clinically relevant information, communication and education throughout the continuum of care (Gerteis, Edgman-Levitan, Daley, \& Delbanco, 1993). In addition, this notion of patient-centred care incorporates patient preferences for the delivery of care and stresses the importance of coordination and of integrated care. Patients often fear that they are not being given all of the information about their illness or prognosis (Gerteis et al., 1993). For cancer patients, the need for information continues to exist despite the plethora of information now available on the internet and through other resources and programs (CCS, 2003).

Several studies have identified the potential of harnessing new technologies that provide patients with secure access to their electronic health record to address many of these aspects of care, including patient empowerment, increased satisfaction, better patientprovider relations and improved continuity of care (Pyper, Amery, Watson, \& Crook, 2004; Ueckert, Goerz, Ataian, Tessmann, \& Prokosch, 2003; Winkelman \& Leonard, 2004).

It is well established that many patients are interested in viewing their medical records (Pyper et al., 2004; Ross et al., 2005). In a published study of 4,500 patients surveyed in $2001,77 \%$ of the participants indicated that they were either somewhat or very interested in reading their clinical record (Fowles et al., 2004). Patients are interested in accessing their records for a number of different personal reasons ranging from a desire for greater understanding of their disease to improved communication and an increased sense of control (Ross et al., 2005). Studies indicate that the greatest predictors of a patient's desire to read the medical record are the number of benefits that they perceive in accessing the records and their information-seeking behaviours, including internet usage, rather than clinical characteristics or educational levels (Fowles et al., 2004; Ross et al., 2005).

The range of benefits of providing patients with access to their records is still somewhat undefined (Ross \& Lin, 2003). Although most studies identify the need for further research in this area, there is some evidence of an increase in patient satisfaction and improved patient compliance with self-care (Ross, 2004; Winkelman \& Leonard, 2004). Research also suggests that making electronic health records accessible to patients may empower them (Ralston, Revere, Robins, \& Goldberg, 2004; Ueckert et al., 2003). A recent study from Britain indicated that patients find it useful to have access to their health records, but they want to be included in the process of developing the system for patient access (Pyper et al., 2004). A randomized control study of patients with congestive heart failure demonstrated that providing patients with online access to their record is feasible and improves adherence, although no effect on health status could be demonstrated (Ross, 2004). This area of research is still in its infancy and many issues still require extensive study (Ralston et al., 2004; Winkelman, 2004).

In the last few years, several different systems for providing patients with access to their electronic health records (EHR) have been developed and tested. These systems include PCASSO (Masys, Baker, Butros, \& Cowles, 2002), PatCIS (Cimino, Patel, \& Kushniruk, 2002) and SPPARO (Ross, 2004). All of these technology studies have demonstrated the ability to provide patients with access to their records in a secure and confidential manner (Cimino et al., 2000; Ueckert et al., 2003). Nevertheless, many patients and health care professionals still have concerns regarding security and confidentiality (Fowles et al., 2004; Masys et al., 2002). Furthermore, evidence about whether patients would like to access their records over the internet is not yet

David Wiljer, PhD, Director, Knowledge Management and Innovation, Princess Margaret Hospital, University Health Network, Assistant Professor, Department of Radiation Oncology, University of Toronto

Sima Bogomilsky, RN, BScN, CON $(C)$, Nurse Manager, Princess Margaret Hospital, University Health Network

Pamela Catton, MD, MHPE, FRCPC, Director, Cancer Education Program, Princess Margaret Hospital, University Health Network

Cindy Murray, RN, MN, Advanced Practice Nurse, Princess Margaret Hospital, University Health Network

Janice Stewart, RN, BScN, CON(C) Nurse Manager, Princess Margaret Hospital, University Health Network

Mark Minden MD, PhD, FRCPC, Princess Margaret Hospital, University Health Network, Professor, Department of Medical Oncology, University of Toronto

Contact Information for correspondence or reprint requests: David Wiljer,PhD, David.Wiljer@uhn.on.ca 
conclusive, with at least one study demonstrating a strong polarity between patients who would and who would not want the ability to access their records online (Fowles et al., 2004; Ross et al., 2005).

For the patients who do want to access their records online, little is known about how and when they would like to access their records, what they would like to see, and what support they would require to access and understand their electronic health record. The sparse literature that does exist indicates that patients would primarily use the record to access laboratory results and physician notes (Cimino, Patel, \& Kushniruk, 2001; Fowles et al., 2004). Several studies also indicate that patients accessing their clinical data should be provided with educational material that will help them understand and interpret what they are viewing (Abidi, Han, \& Abidi, 2001; Doupi \& van der Lei, 2002; Pyper et al., 2004). Research suggests tailoring education for individual patients improves the patient experience and ensures that patients receive the information that is relevant to them (Ziebland et al., 2004). There has also been a significant trend toward providing tailored and personalized patient information to empower and support patients during their clinical experiences (Abidi et al., 2001). The electronic health record provides a unique opportunity to provide tailored information that is clinically relevant and to promote and support self-managed care (Winkelman \& Leonard, 2004).

The potential benefits of providing patients with access to their health record extends beyond education and has enormous potential to deliver patient-centred care systems that could improve the patient experience and impact positively on health outcomes (Winkelman \& Leonard, 2004). However, patient access to elements of their electronic medical record such as test results has the potential to cause distress to patients and staff (Pyper et al., 2004). Although no adverse events have been reported in the literature, health care professionals remain concerned that patients could face several challenges if they are provided clinical results online. These perceived challenges by physicians, for example, include patient confusion when reading lab results or x-ray reports, an increase in patient anxiety or patient worry, and an increase in the number of questions being asked (Ross et al., 2005). There is, however, a paucity of empirical data to weigh the potential benefits for patients and the perceived risks of health care professionals.

In investigating how new technologies can support patient-centred care, the malignant hematology patient population at Princess Margaret Hospital/University Health Network was identified as an appropriate target population for this needs assessment and pilot study. The patient volumes are high ( $n=400-500$ per week), clinic visits often last an entire day, treatment occurs over a long period of time (from several months to three years) and requires frequent visits to the clinic. The patient population is very diverse, including both sexes, wide age ranges, and varied cultural and educational backgrounds. Anecdotally, staff and patients had expressed interest in providing patients with access to their clinically relevant information to improve the overall patient experience.

\section{Purpose}

The purpose of this study is to conduct a needs assessment for providing hematology oncology patients access to elements of their electronic health record. The assessment will help to identify the attitudes, preferences and needs of patients, and validate and explore themes and concepts identified in the literature. The results from this needs assessment will form the basis for the development of a working prototype for a future pilot project that will study the feasibility of providing patients access to elements of their own electronic health record and tailored health information that is clinically relevant.

\section{Methods}

This study has three distinct elements. First, a patient questionnaire was developed and administered to identify patient needs and preferences for utilizing the internet for obtaining clinical information from their record. Secondly, opened-ended surveys were conducted to explore staff perceptions about providing patients access to their electronic health record. Finally, based on the data gathered throughout this study, a Web-based prototype was constructed in order to conduct a pilot study that will examine the feasibility of this initiative.

\section{Patient Questionnaire}

A patient questionnaire was developed for malignant hematology patients at Princess Margaret Hospital. The closed-response questionnaire consists of 21 factual questions regarding the participants' use of the internet and their interest in using the internet to obtain personal health information, including access to selected items of their electronic health record; patients were also asked demographic questions and whether or not they would be willing to participate in a pilot study to access elements of their electronic health record over the internet. The survey took 20 to 30 minutes to complete and eligible patients had hematological malignancies and had to be conversant in English.

An advanced practice nurse (APN) in the transfusion unit and leukemia outpatient clinics at Princess Margaret Hospital recruited patients and administered the questionnaire over a one-month period. Patients waiting in the hematology clinic and transfusion clinics were approached by an APN, the study was explained to them and consent obtained; they were then given copies of the survey. The survey took 20 to 30 minutes to complete. During this period, 46 patients consented and completed the questionnaire. Data from the completed surveys were entered into an online survey tool, data were checked for accuracy and frequencies were generated. Due to the small number of participants, only descriptive statistical analysis techniques were employed.

\section{Staff Interviews}

A research assistant administered a questionnaire to clinical staff members directly involved with the malignant hematology patient clinic. The open-ended questionnaire was constructed to validate the concerns and issues identified by the research team through the literature review, patient survey and analysis of the current clinical systems and processes. The questionnaire included five demographic questions, and then participants were asked to comment on how patient access to their health records would impact on the clinical experiences. Participants were asked to comment on content areas and related topics identified through the literature and informal discussions with clinicians. The questionnaire probed the following content areas: 1) the challenges of providing patient access to their records; 2) the patient need for education and clear communication; 3 ) the potential for increased patient self-care and 4) the potential impact on staff workload. Participants were selected based on a quota sample to include a range of disciplines. An independent research assistant who was not a member of the clinical staff or research team administered the questionnaire. The opened-ended questionnaire took about 45 minutes to complete and each session was audio-recorded. The demographic results were entered into a spreadsheet and frequencies were generated; members of the research team summarized participant comments in each of the content areas.

\section{Prototype Development}

Based on the results of the needs assessment, a prototype was designed for providing patients with access to their electronic health record. Key elements of the health record were selected based on the needs assessment. The Shared Information Management Services (SIMS) Department at the University Health Network developed the prototype. The project architecture was planned to protect patient information and provide the information in a way that patients could easily understand.

All aspects of the study protocol and development of the prototype were reviewed and approved by the University Health Network Research Ethics Board. 


\section{Results}

\section{Patient Survey}

Forty-six malignant hematology patients, 30 males and 16 females, were surveyed. The majority of participants (71\%) identified English as their first language. The majority of patients were between 21 and 60 years of age (See Table 1). All of the respondents had heard of the internet and $89 \%$ of the participants access the internet from home, $18 \%$ from work, and $18 \%$ through a friend or family member, $11 \%$ from clinic/hospital and $11 \%$ from the library.

Sixty-one percent of the participants reported using the internet to access health information online. Eighty-nine percent of the participants were interested in using the internet to access their electronic health record or chart. $87 \%$ expressed a desire to use the internet to obtain laboratory results, including blood tests (See Table 2). Eighty percent of the participants stated that they would regularly check their blood tests and $79 \%$ of the participants stated that they would require educational materials in order to understand online test results and to know what to do about these results. The majority of participants also stated that they would like to use the internet to access online patient education materials and consult with a hospital health care professional about non-urgent matters.

The majority of the participants $(87 \%)$ would like to have access to their personal registration data. Eighty-two percent of the participants stated that they would like the ability to make changes to their own registration data, such as date of birth and contact information for emergencies, family and referring physician, as well as diagnostic information: $43 \%$ stated they would like to make changes to a computer form; $9 \%$ would like to make changes to a paper form; and 34\% would like to make changes to both the computer and paper forms.

\section{Staff Interviews}

Seven staff members participated in the study. There were five female and two male participants ranging in age from 36 to 58. The participants represented a range of disciplines and clinical experience. There were four nurses, one physician, one patient flow co-ordinator and one administrative assistant. Two participants reported having fewer than five years of clinical experience; two reported five to 10 ; and three reported having more than 10 years of clinical experience.

\begin{tabular}{|l|l|l|}
\hline \multicolumn{2}{|l|}{ Table 1: Population Characteristics } & $\%$ \\
\hline Characteristics & $\mathrm{n}$ & \\
\hline Sex (n=46) & & 65.2 \\
\hline Male & 30 & 34.8 \\
\hline Female & 16 & \\
\hline Age (n=46) & & 10.9 \\
\hline Under 21 & 5 & 34.8 \\
\hline $21-40$ & 16 & 30.4 \\
\hline $41-59$ & 14 & 23.9 \\
\hline $60+$ & 11 & \\
\hline Education (n=45) & & 0 \\
\hline Elementary & 0 & 33.3 \\
\hline High school & 15 & 42.2 \\
\hline Undergraduate/College & 19 & 24.4 \\
\hline Post-graduate & 11 & \\
\hline $\begin{array}{l}\text { Note: Percentages that do not add to 100\% are due to missing } \\
\text { values and rounding. }\end{array}$ & \\
\hline
\end{tabular}

The results confirmed that the challenges of providing patients access to their records centred around issues of confidentiality and the potential for records to be accessed by unauthorized people if passwords were not adequately protected. Staff identified potential barriers including access to computers, low levels of computer literacy among elderly patients, language barriers and high levels of confusion and anxiety. Some staff members expressed concerns about patients having access to all of the items in the record. All participants stated that patients should have access to their blood results. There was no consensus about access to information that could be confusing to patients, such as imaging results, physician notes, and pathology reports. Some of the participants thought patients should have access to blood results as soon as they are available, but others thought the results should become available during office hours so that questions could be answered more readily.

Staff agreed that education and communication issues needed to be addressed prior to conducting the pilot study. Staff expressed the opinion that patients should be adequately supported by trained staff

Table 2: Would you like to, either by yourself or by someone close to you, access any of the following using the internet: $(n=46)$

\begin{tabular}{|c|c|c|}
\hline & $\mathrm{n}$ & $\%$ \\
\hline \multicolumn{3}{|c|}{ Selected Items in the Chart } \\
\hline Yes & 41 & 89.1 \\
\hline No & 1 & 2.2 \\
\hline Unsure & 4 & 8.7 \\
\hline \multicolumn{3}{|c|}{$\begin{array}{l}\text { Access and Review } \\
\text { Registration Data }\end{array}$} \\
\hline Yes & 40 & 87.0 \\
\hline No & 1 & 2.2 \\
\hline Unsure & 5 & 10.8 \\
\hline \multicolumn{3}{|c|}{ Laboratory Reports } \\
\hline Yes & 40 & 87.0 \\
\hline No & 2 & 4.3 \\
\hline Unsure & 4 & 8.7 \\
\hline \multicolumn{3}{|c|}{ Radiology Reports } \\
\hline Yes & 39 & 84.9 \\
\hline No & 2 & 4.3 \\
\hline Unsure & 5 & 10.8 \\
\hline \multicolumn{3}{|c|}{ Patient Education Material } \\
\hline Yes & 42 & 91.3 \\
\hline No & 2 & 4.3 \\
\hline Unsure & 2 & 4.3 \\
\hline \multicolumn{3}{|c|}{$\begin{array}{l}\text { Non-Urgent Consultation } \\
\text { with Health Care Professional }\end{array}$} \\
\hline Yes & 40 & 87.0 \\
\hline No & 1 & 2.2 \\
\hline Unsure & 5 & 10.8 \\
\hline \multicolumn{3}{|c|}{$\begin{array}{l}\text { Note: Percentages that do not add to } 100 \% \text { are due to missing } \\
\text { values and rounding. }\end{array}$} \\
\hline
\end{tabular}


members, given face-to-face training and that the materials and training needed to be multilingual. The need to educate patients about how to interpret their health record by providing information about the disease and test results, such as blood values, was widely confirmed and some members also indicated that staff members should be available to answer patient questions.

Staff thought that providing patients with access to their health records would increase participation in the decision-making process and give them more control, thus increasing the potential for patient self-care. Staff members underlined the importance of allowing patients the choice of self-care. The majority of staff believed younger patients would benefit more from being given access to their health record.

Finally, staff thought that this initiative could initially increase the number of questions they receive and that there was a potential for an increase in patient confusion, but ultimately thought this could decrease the workload and improve the patient experience. Despite all of the potential barriers and concerns that were discussed, all of the participating staff stated that the benefits of providing patients with access to their record would outweigh the challenges that need to be addressed.

\section{Staff Perceptions}

Potential Benefits:

- Decrease workload

- Improved work flow

- Better management of patients

- Decrease in unnecessary hospital visits

Potential Challenges

- Integration of new technology with existing systems

- Resistance to changes in the system

- Providing adequate support and education

- Increase in time required to manage patients and more questions/ phone calls

- Increase risks of breaches in security and confidentiality

\section{Prototype Development}

Four key areas were identified for prototype development based on the needs assessment: 1) a secure login, 2) access to registration data, 3 ) current blood test results and 4) education to help patients interpret their results and contact their treatment team, if necessary. This webbased application has been designed to provide patients with an easyto-use, clear and concise interface display. The information has been drawn from the electronic health record and is displayed in this interface. Patients will also be able to print the results easily. Patients will be provided with plain-language information on how to interpret the results and contact a health care provider. In addition, education will be provided in a number of multimedia formats, including video clips of physicians explaining the importance of the results, how to interpret them, what to do based on these results, and whom they should contact. The prototype is currently being used to conduct a pilot study in the clinical environment.

\section{Discussion}

The majority of patients are interested in using the internet as a vehicle for improving the clinical experience and the clinical encounter. Patients are searching for health information online, but what is important to note from this study is the type of information that patients would like to find online. Patients do not just want to search for general health information on the internet. They also want to access information that is clinically relevant to their own condition or health status. They want to access their hospital registration data, their lab reports and their radiology reports. In addition, they want to use the internet as a mechanism for contacting their health care professionals regarding non-urgent clinical issues or concerns.
This particular study did not demonstrate the polarity that other studies have reflected between those patients who would like access to their electronic health record online and those who do not want their records available online. Other patient populations have expressed interest in viewing their health records, but they have been typically split on whether or not the results should be distributed and accessed online (Fowles et al., 2004; Ross et al., 2005) . However, this particular study group was not divided on the issue of online access; in fact, it was overwhelmingly in favour of receiving results online. This study may reflect changing attitudes to online access to sensitive and personal information, but more probably this difference can be attributed to the type of patient population being surveyed. This patient population has very specific clinical needs. Malignant hematology patients come for frequent clinical appointments, they spend long hours in the clinic and are typically well educated about their own health condition. The proposed approach of providing online access to elements of the electronic health record may help this patient population manage better throughout treatment.

The results reported in this study did not probe deeply into the perceived benefits of online access for patients since these issues will be explored more fully in the proposed pilot study. Nevertheless, it is clear from the literature that providing patients with access to their electronic health record could potentially empower them to participate in their care in a number of important ways. Patients could participate in ensuring that their personal information is accurate and up-to-date and the majority of participants in this study were interested in viewing and updating their information. The ability to keep information up to date could in fact improve the continuity of care. Since many EHR systems utilize an auto-fax or mailing process to send records to referring and family physicians, this would provide patients with the opportunity to ensure and verify that medical information is being distributed in a timely and accurate manner between members of their health care team.

In addition to viewing personal information, patients who have access to their EHR can monitor their test results, including blood test results. This particular study population requires frequent blood tests, and careful monitoring of these results is an important aspect of their care. Allowing patients to access their own results could potentially reduce the time patients spend in the hospital waiting for results and give them an improved sense of control over their situation. It may also lead to increased satisfaction with their care or better quality of life. Access to the health record could also potentially facilitate using the internet for non-urgent communications between patients and their health care providers (Eysenbach \& Jadad, 2001; Jadad \& Delamothe, 2004). If patients can communicate via the internet on non-urgent matters, it could reduce the number of times a patient has to come to the clinic. By integrating educational material into the record, there is also a unique opportunity to provide tailored, personalized information that is current, reliable and relevant to a patient's situation. The proposed pilot study will afford the opportunity to explore these potential benefits further.

There is, however, a professional responsibility for everyone involved in the development of this process to ensure all potentially harmful consequences have been considered and that appropriate educational materials and programs are developed to help people understand the information they are given. As both patients and health care providers have indicated, access to the record must be delivered in a secure, confidential environment that includes an educational component to help patients understand their health record. Providing patients with access to their records may require shifts in clinical practice that extend beyond providing patients with tailored information. For example, current research is not conclusive about the best way to provide information that could potentially be "bad news" or confusing, although limited research indicates that bad news may need to be managed in a face-to-face encounter or consultation (Pyper et al., 2004). Certain types of results, therefore, may have to be monitored by the primary care team before patients have access to them. 
The benefits must be weighed against the potential risks and a number of questions need to be answered. The clinical experience in this area is somewhat limited (Hassol et al., 2004; Pyper et al., 2004; Ross, 2004) and there are far more questions that are emerging than answers to particular questions and concerns. Clearly, however, this is an issue that will not disappear. As more organizations move to an electronic health record and patients become more comfortable with accessing their health information online, there will be a growing pressure to provide a whole range of results online. Therefore, a great deal more research is required in this area of clinical care.

\section{Limitations}

This study focuses on a group of patients who are typically active participants in their care. The sample size is small and patients in this population visit the clinic frequently throughout the course of their treatment and are typically very involved and well informed about their condition. The research was completed in Canada with fully insured and accessible health care and the results may not be applicable in other health systems. The applicability of these data to patients with other types of cancer and other chronic illnesses should be explored more fully.

\section{Conclusions and Future Directions}

Hematology oncology patients are searching for health information on the internet, but general information may not be sufficient for many of these patients. Patients who participated in this study are more interested in using the internet to monitor their clinical information and communicate with their health care team than they are in finding health information. In addition, patients expressed a strong interest in participating in their care by viewing the data in the electronic health record. Although staff raised many concerns and potential challenges, they believe that the benefits of improving the patient experience, of supporting self-care and improving workflow and workload outweighed the short-term challenges. Based on the data gathered through this study, a Web-Based prototype to provide patients with the ability to review their registration data, blood results and tailored information has been developed for pilot testing. This prototype will allow us to conduct further research around the issues of empowering patients to participate in their care through only access to their electronic health records.

\section{Acknowledgements}

We would like to thank Matt Anderson, Lydia Lee and members of the Shared Information Management Services (SIMS) Department at the University Health Network who provided the leadership and resources to develop and support the hematology portal throughout this study. We would also like to thank Vivian Cornelius and Erin Jones for their research support on this project, as well as the patients and staff who generously contributed their time.

Jadad, A. R., \& Delamothe, T. (2004). What next for electronic communication and health care? BMJ, 328(7449), 1143-1144.

Masys, D., Baker, D., Butros, A., \& Cowles, K. E. (2002). Giving patients access to their medical records via the internet: the PCASSO experience. J Am Med Inform Assoc, 9(2), 181-191.

Pyper, C., Amery, J., Watson, M., \& Crook, C. (2004). Patients' experiences when accessing their on-line electronic patient records in primary care. Br J Gen Pract, 54(498), 38-43.

Ralston, J. D., Revere, D., Robins, L. S., \& Goldberg, H. I. (2004). Patients' experience with a diabetes support programme based on an interactive electronic medical record: qualitative study. BMJ, 328(7449), 1159.

Ross, S. (2004). Providing a web-based online medical record with electronic communication capabilities to patients with congestive heart failure: Randomized trial. J Med Internet Res, 6(2), e12.

Ross, S., Todd, J., Moore, L. A., Beaty, B. L., Wittevrongel, L., \& Lin, C. T. (2005). Expectations of patients and physicians regarding patient-accessible medical records. J Med Internet Res, 7(2), e13.

Ross, S. E., \& Lin, C. T. (2003). The effects of promoting patient access to medical records: a review. J Am Med Inform Assoc, 10(2), 129-138.

Ueckert, F., Goerz, M., Ataian, M., Tessmann, S., \& Prokosch, H. U. (2003). Empowerment of patients and communication with health care professionals through an electronic health record. Int J Med Inf, 70(2-3), 99-108.

Winkelman, W. (2004). Reconciling the Patient's Role in the Improvement of Health Outcomes: Medical Informatics' Newest Frontier. Journal of Medical Internet Research, 6(2):e14.

Winkelman, W. J., \& Leonard, K. J. (2004). Overcoming structural constraints to patient utilization of electronic medical records: a critical review and proposal for an evaluation framework. J Am Med Inform Assoc, 11(2), 151-161.

Ziebland, S., Chapple, A., Dumelow, C., Evans, J., Prinjha, S., \& Rozmovits, L. (2004). How the internet affects patients' experience of cancer: a qualitative study. BMJ, 328(7439), 564. 